\title{
ALTERAÇÃO DA COMUNIDADE ZOOPLANCTÔNICA DO SACO DOS LIMÕES APÓS IMPACTO DAS OBRAS DA VIA EXPRESSA SUL - BAÍA SUL DA ILHA DE SANTA CATARINA
}

\author{
VEADO, L.D. Ad V. \& RESGALLA Jr., C. \\ CTTMar/UNIVALI - Caixa Postal 360, Itajaí, SC \\ ludmillaveado@ig.com.br; cresgalla@univali.br
}

\begin{abstract}
Veado, L.D. Ad V. \& Resgalla Jr., C. 2005. Zooplankton community change after impact of the Via Expressa Sul works - Southern Bay of Santa Catarina Island. Braz. J. Aquat. Sci. Technol. 9(2):65-73. ISSN 1808-7035. In order to solve traffic problems in the capital, the government of the Santa Catarina State began in 1996 dredging works and hydraulic embankment in the "Saco dos Limões", south bay of Santa Catarina Island. Studies of environmental impact accomplished by CTTMar (1996/1997) verified that the dredging activities altered the community of the local zooplankton. The present work studied the zooplankton community 6 years after the end of the dredging activities, diagnosing the possible alterations in the environment. The zooplankton samples were obtained between 1996 and 1998 (Phase 1, impact) and from 1999 to 2002 (Phase 2, after impact) by superficial hauls using a net WP-2 type with $200 \mathrm{~mm}$ of mesh size. The community's typical species found were the copepods Acartia lilljeborgi, A. tonsa, Oithona oswaldocruzii and Paracalanus quasimodo. Among the two phases a significant decline was observed in the density of the zooplankton. $A$. tonsa, typical estuarine copepod was substituted by $A$. lilljeborgi inside of the bay. The same was observed for $P$. quasimodo that substituted $O$. oswaldocruzii in the intern area. These results suggest the hydrological changes in function of the increase of the depth, decrease of the area and the residence time of the water, impeding the development of estuarine community that was characteristic before the dredging and hydraulic embankment activities.
\end{abstract}

Keywords: Zooplankton, Environmental impact, Dredging, Hydraulic embankment, Santa Catarina, Brasil

\section{INTRODUÇÃO}

A enseada do Saco dos Limões, situada na margem leste da Baía Sul da Ilha de Santa Catarina (Florianópolis - SC), é caracterizada como sendo um ambiente estuarino de pouca profundidade, de baixa energia hidrodinâmica e com deposição de lama terrígena numa extensa planície intermareal, que sofre influência do rio Tavares. Essas condições físicas, somadas à ocorrência de larvas meroplanctônicas (ENGEVIX, 1996), apontaram o ambiente como berçário de espécies de crustáceos e peixes (Abreu et al.,1998).

O ecossistema apresenta duas áreas de interesse conservacionista: a Reserva Extrativista Marinha do Pirajubaé, com a exploração do berbigão Anomalocardia brasiliana, e o manguezal do rio Tavares. A presença destas regiões leva a considerar o Saco dos Limões como um ambiente de importâncias sócioeconômica e de conservação.

Em 1996, o governo do Estado de Santa Catarina iniciou a construção da Via Expressa SC-Sul, às margens da Baía Sul. O projeto constou na retirada de 8,5 $\mathrm{x} 10^{6} \mathrm{~m}^{3}$ de areia, obtida em uma jazida no próprio ambiente, para a construção de um aterro de $15,9 \mathrm{~km}$ de extensão (Abreu et al., 1998). As atividades de dragagem e aterro no ambiente tiveram início em agosto de 1996 e, com exceção de um levantamento reali- zado em outubro de 1995 (ENGEVIX, 1996), os trabaIhos de diagnóstico e monitoramento dos prováveis impactos foram realizados durante e logo após as atividades de dragagem (1996 a 1998).

Os resultados apresentados em Resgalla Jr. (2001), durante o período de dragagem, mostraram que a comunidade zooplanctônica é diversa, abundante e com uma nítida sazonalidade quali-quantitativa. Os impactos causados pelas atividades de dragagem e aterro se estenderam desde fatores físicos, como a redução da área, até fatores alimentares influenciando a produção de ovos e náuplios por Acartia tonsa e $A$. lilljeborgi.

O aumento da concentração do material em suspensão (MS) na coluna d'água, durante as atividades da dragagem, desequilibrou a cadeia trófica local. Segundo Resgalla Jr. (2001), em janeiro de 1997 a alta concentração do MS, mas de baixo conteúdo orgânico, pôde ter acarretado uma deficiência metabólica aos organismos, alterando as taxas de sobrevivência e recrutamento, o que pode explicar as baixas densidades dos copépodos Acartia tonsa e A. lilljeborgi que são típicos de regiões estuarinas e costeiras. Outro impacto promovido pela alta carga de material em suspensão poderia ser atribuído à ação de metais que foram biodisponibilizados para os organismos quando ressuspendidos do fundo. 
Segundo as exigências da Procuradoria do Estado, o monitoramento ambiental constou de dois períodos amostrais, um durante e outro após os impactos promovidos pelas atividades de dragagem na região do Saco dos Limões (Baía Sul - Florianópolis). O primeiro período iniciou em outubro de 1996 e finalizou em janeiro de 1998. O segundo iniciou em julho de 1999, e estendeu-se até janeiro de 2002. Entre os vários programas do monitoramento, a segunda fase dos estudos constou na avaliação quali-quantitativa da comunidade zooplanctônica do Saco dos Limões, após ter sofrido o impacto da dragagem. Para isso, foram comparadas as densidades médias do zooplâncton total entre os períodos de impacto e após impacto e as variações de densidade dos principais grupos zooplanctônicos. Neste estudo, procurou estabelecer uma possível espécie indicadora do impacto ocorrido no ambiente e, finalmente, um diagnóstico da situação atual do ambiente quanto à comunidade zooplanctônica, frente ao impacto ocorrido em 1996/1997.

\section{MATERIAL E MÉTODOS}

As coletas das amostras de zooplâncton foram realizadas trimestralmente entre 1999 e 2002 (Fase 2 - pós impacto) em 6 pontos distribuídos ao longo do saco dos Limões. O ponto \#1 localizou-se no canal da Baía Sul, indicando o aporte de água costeira, a fonte principal de organismos zooplanctônicos, que estariam disponíveis a colonizar o ambiente. Os pontos \#2 e \#6 foram considerados como a comunidade que representaria os limites do saco dos Limões e os pontos \#3, \#4 e \#5, as comunidades que apresentaram maior ca- pacidade de sobreviver em condições bióticas e abióticas do local, cuja água é influenciada pelo aporte do rio Tavares (Fig. 1).

Os arrastos de zooplâncton foram realizados com uma rede tipo WP-2 de 0,2 mm de tamanho de malha, $30 \mathrm{~cm}$ de diâmetro de boca e equipada com um fluxômetro. Os arrastos foram executados de forma oblíqua, integrando toda a coluna de água nos pontos de maior profundidade (\#1, \#2 e \#6) e de forma horizontal de superfície nos pontos de menor profundidade (\#3, \#4 e \#5). Após a coleta, as amostras foram imediatamente fixadas em solução de formalina a $4 \%$.

Dados de temperatura, salinidade e transparência da água foram também obtidos segundo a metodologia apresentada por Abreu et al. (1998).

Em laboratório, as análises qualitativas e quantitativas foram realizadas ao menor táxon possível, utilizando microscópio estereoscópio, microscópio biológico e câmaras do tipo Bogorov. O procedimento recomendado por Boltovskoy (1981a) constou no fracionamento das amostras para contagens com subamostradores do tipo pistão, perfazendo no mínimo $10 \%$ da amostra total. Somado a isto foi realizada uma revisão total da amostra para registro de espécies em baixas densidades. Para as classificações das espécies foram utilizados os trabalhos do Boltovskoy (1981b, 1999) e Montú \& Gloeden (1986).

Os resultados das análises quali-quantitativas foram expressos em número de organismo por metro cúbico de água filtrada pela rede. O índice de Riqueza de Margalef foi calculado segundo Omori \& Ikeda (1984), onde:

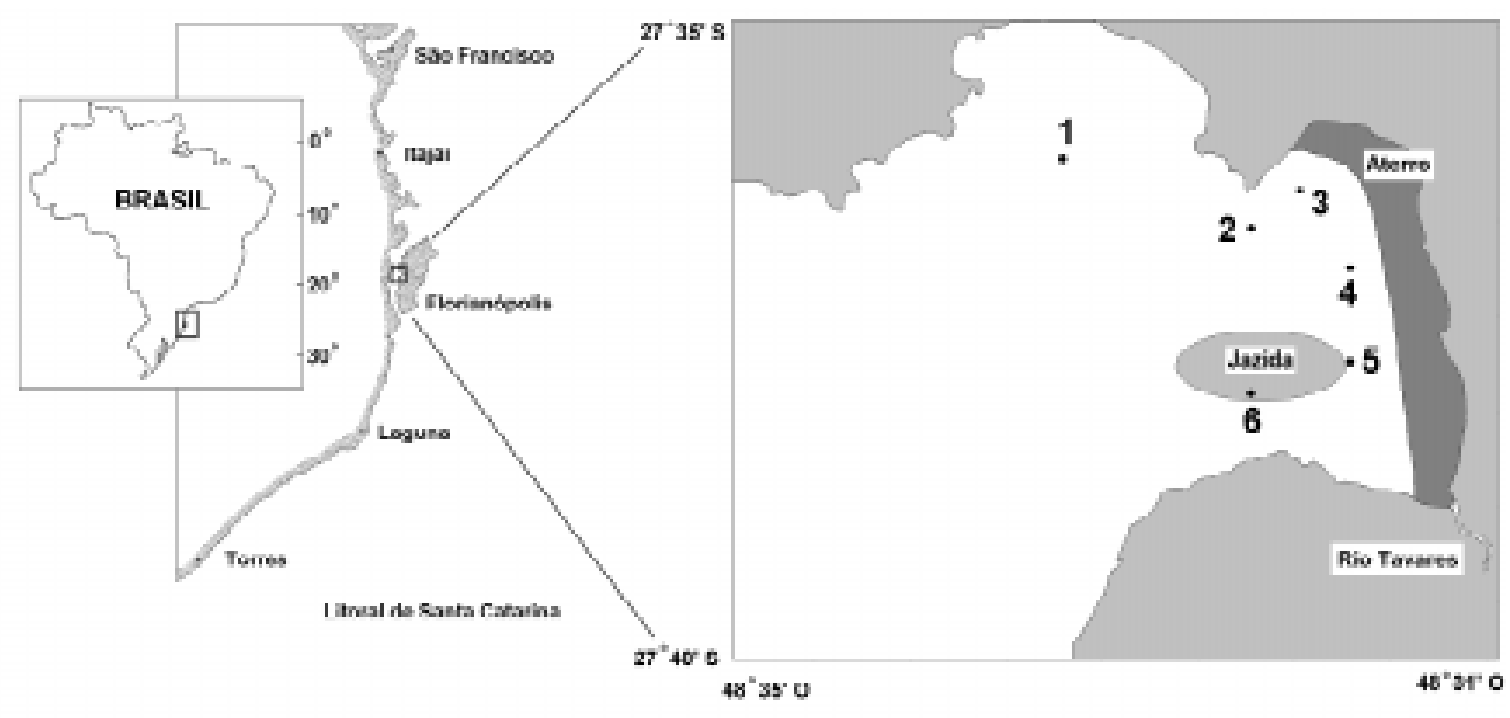

Figura 1 - Localização do saco dos Limões, Baía Sul da llha de Santa Catarina e os 6 pontos de coleta de zooplâncton. 


$$
D=\frac{S-1}{\operatorname{Ln}(N)}
$$

Sendo:

$\mathrm{D}=$ riqueza

$\mathrm{S}=$ número de espécies

$\mathrm{N}$ = número total de organismos (org. $\left./ \mathrm{m}^{3}\right)$

Os dados do zooplâncton da primeira fase (1996 a 1998) foram obtidos de Resgalla Jr. (2001) e referemse ao período durante e logo após o impacto. Para a análise do estado atual do ambiente, os dados foram tratados em dois grupos de pontos amostrais: externo (pontos \#1, \#2 e \#6) e interno a enseada (\#3, \#4 e \#5).

\section{RESULTADOS}

\section{Fatores Abióticos}

Infelizmente devido à descontinuidade da obtenção dos dados abióticos, as informações disponíveis apresentaram limitações na interpretação da variabilidade do zooplâncton. De qualquer forma, foi observado, para a temperatura, uma sazonalidade com maiores valores nos meses de verão e outono $\left(26,9^{\circ} \mathrm{C}\right)$ e menores nos meses de inverno e primavera $\left(17,7^{\circ} \mathrm{C}\right)$. Os maiores valores de temperatura ocorreram nos pontos internos da enseada, mas a amplitude de variação dentro e fora da enseada diminuiu para as amostras obtidas entre 1999 a 2002 (Fig. 2A).

A salinidade (Fig. 2B) apresentou-se mais ou menos constante durante o período amostral com os menores valores dentro da enseada. Entretanto, observou-se que a amplitude de variação dentro e fora do ambiente diminuiu entre a primeira e segunda fase do monitoramento. Este fato indica uma diminuição do gradiente de salinidade dentro da enseada, o que sugere uma maior similaridade com a água costeira.

Para a profundidade do disco de Secchi (ou transparência) os menores valores foram registrados nos meses de primavera e os maiores no verão. A partir do ano de 1999, observou-se pouca diferença das águas dentro e fora da enseada (Fig. 2C).

\section{Zooplâncton}

Para a densidade total dos organismos, os resultados mostraram uma sazonalidade do zooplâncton no ambiente, ou seja, baixas densidades foram observadas nos meses de inverno $\left(160 \mathrm{org} / \mathrm{m}^{3}\right)$ e altas nos meses de verão (3500 org. $/ \mathrm{m}^{3}$ ). Entretanto, a comunidade zooplanctônica mostrou um declínio ao longo dos anos, sendo bem evidente a partir das amostras obti-
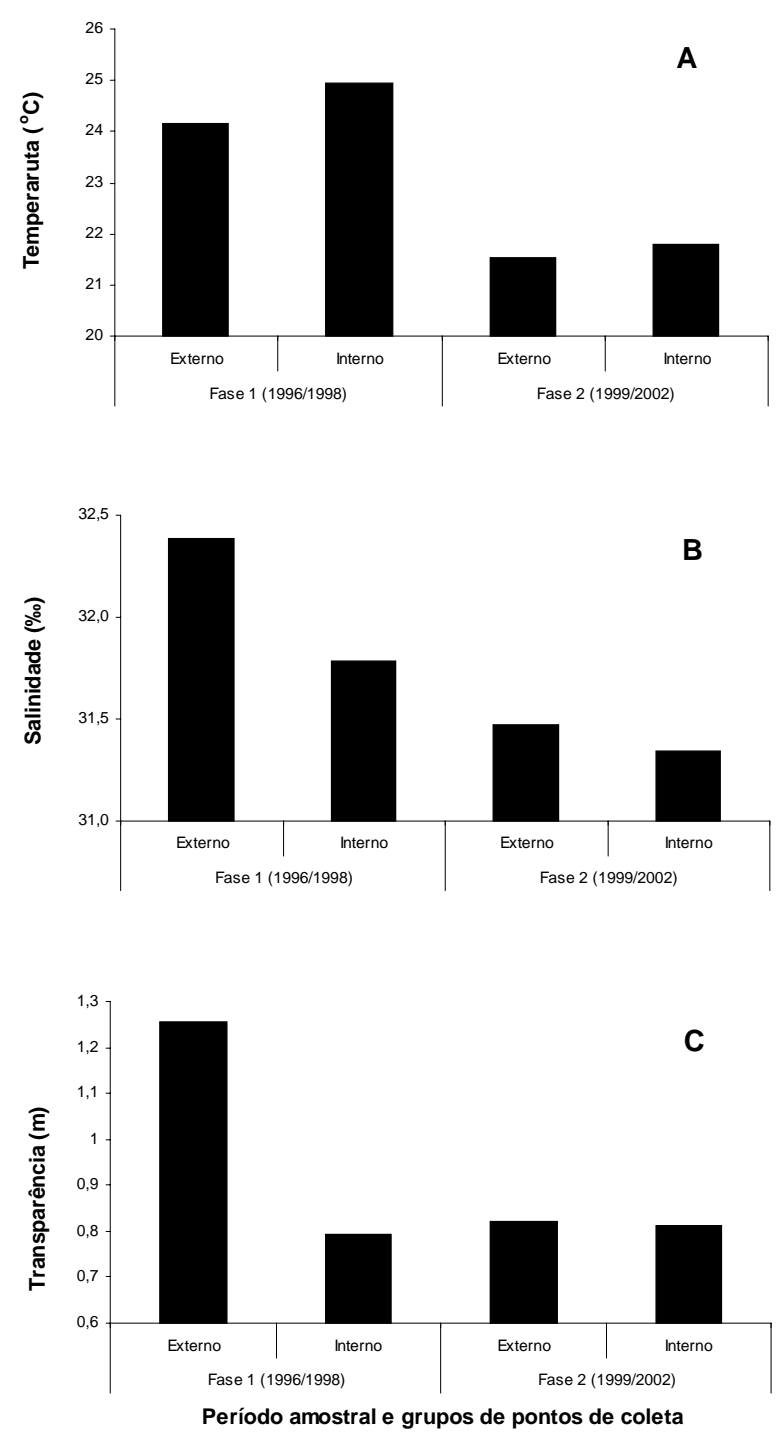

Figura 2 - Valores médios da A) Temperatura $\left({ }^{\circ} \mathrm{C}\right)$, B) Salinidade (\%) e C) Transparência (profundidade do disco de Secchi em metros) agrupados por pontos de coleta externo e interno da enseada e por fase de estudo (Fase 1 - Impacto, Fase 2 - Pós impacto).

das em 1999. E ao contrário do que foi observado para a fase de impacto, os maiores valores de densidade ocorreram sempre na região interna da enseada (Fig. 3).

Os resultados das análises qualitativas mostraram que a enseada apresenta uma fauna diversificada constituída por 77 taxa distribuídos em grandes grupos, famílias, gêneros e espécies (Tabela 1).

Das espécies típicas do Saco dos Limões, podem ser destacados os copépodos Acartia lilljeborgi , A. tonsa, Oithona oswaldocruzii e Paracalanus quasimodo. Acartia lilljeborgi apresentou um acrésci- 


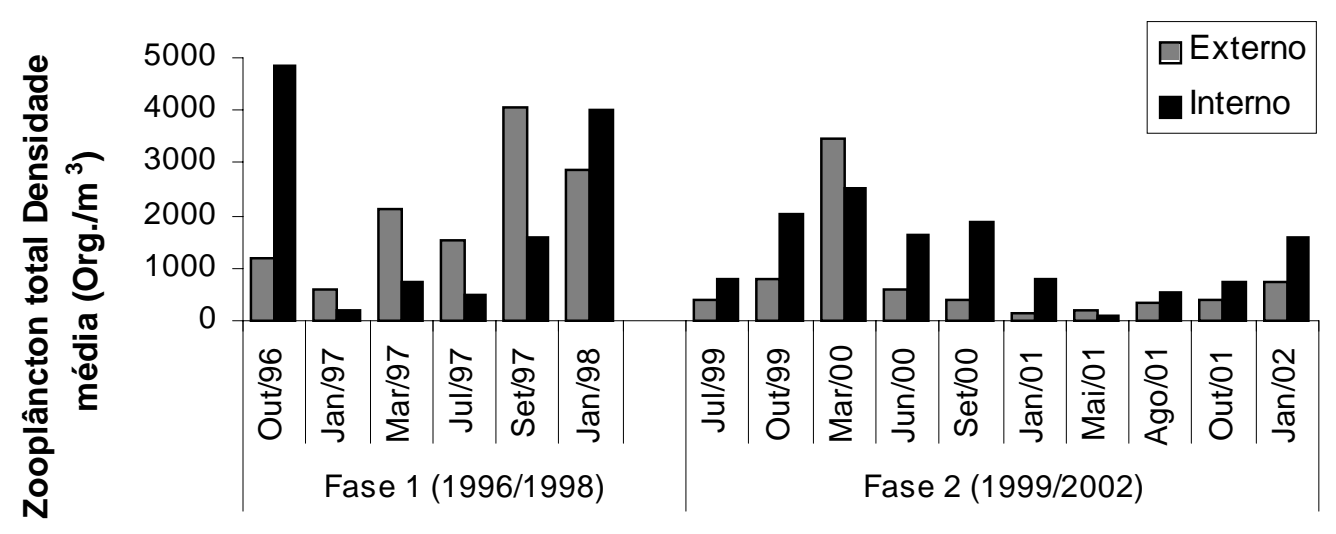

\section{Período amostral e grupos de pontos de coleta}

Figura 3 - Valores médios da densidade total do zooplâncton (Org. $/ \mathrm{m}^{3}$ ) agrupados por pontos de coleta externo e interno da enseada e por fase de estudo (Fase 1 - Impacto, Fase 2 - Pós impacto).

mo na sua densidade ao longo dos anos, e com uma maior invasão na porção interna da enseada (Fig. 4). Por outro lado, $A$. tonsa mostrou um decréscimo na sua densidade acompanhando o comportamento do zooplâncton total, passando a ocupar a região interna da enseada (Fig. 5).

Oithona oswaldocruzii (Fig. 6) e Paracalanus quasimodo (Fig. 7) apresentaram baixas densidades quando comparadas com as espécies de Acartidae. $O$. oswaldocruzii ocorreu de forma aleatória ao longo dos anos e com maior representatividade no limite interno da enseada. Ao contrário, a distribuição de $P$. quasimodo ocorreu, preferencialmente, nos pontos externos com invasão para a parte interna da enseada a partir de 1999.

De maneira geral o índice de riqueza de espécies (Fig. 8) apresentou uma oscilação sazonal com altos valores nos meses quentes e baixos nos frios para as amostras pós-impacto (1999/2002). Comparativamente, houve um aumento na riqueza entre as duas fases de amostragem, com altos índices na região interna da enseada.

\section{DISCUSSÃO}

Devido à descontinuidade na obtenção dos dados abióticos (temperatura, salinidade e transparência), os resultados deste estudo foram interpretados a luz das informações das características do zooplâncton dominante na enseada do Saco dos Limões. Desta forma, os organismos foram utilizados como indicadores hidrológicos, destacando a existência ou não de alterações no ambiente, durante e após as atividades de dragagem e aterro. Entretanto, os valores médios dos parâmetros abióticos sugerem uma maior semelhança entre as águas da região costeira e a do interior da enseada (Fig. 2), o que diferencia os dois períodos de amostragem.

A densidade total dos organismos no Saco dos Limões, apresentou uma sazonalidade bem definida (Fig. 3), o que pode ser considerado um comportamento natural da comunidade zooplanctônica da enseada. Esta sazonalidade já foi destacada por Resgalla Jr. (2001), que também relatou a similaridade específica com a costa da região sudeste no verão e com a costa do Rio Grande do Sul no inverno. No entanto, a densidade total dos organismos apresentou um declínio a partir de julho de 1999.

Os fatores impactantes presentes durante o período de dragagem e aterro, destacados por Abreu et al. (1998) e Resgalla Jr. (2001), foram a biodisponibilização de constituintes tóxicos presentes no sedimento e a alteração na transparência da água dentro da enseada. Entretanto, tais processos dificilmente poderiam permanecer atuando 5 anos após as atividades de dragagem e aterro terem sido concluídas.

A conclusão da dragagem e do aterro no Saco dos Limões, possivelmente, acarretou alterações na hidrodinâmica da região. A enseada sofreu alterações físicas importantes, como uma maior exposição devido à diminuição da sua área (aterro) e um aumento da profundidade média (dragagem de jazida de areia). Essas alterações provavelmente permitiram uma maior circulação da água marinha adjacente e, consequentemente, um menor tempo de residência que podem influenciar o tipo de ocupação das espécies de zooplâncton. Esta suposição é fortemente confirmada pela diminuição nos gradientes dos fatores abióticos observados entre as regiões externa e interna da enseda (Fig. 2). 
Braz. J. Aquat. Sci. Technol., 2005, 9(2):65-73.

Tabela 1 - Grupos do zooplâncton presentes no Saco dos Limões, llha de Santa Catarina (SC) durante o período amostral compreendido entre 1999 a 2002

\begin{tabular}{|c|c|}
\hline Coelenterata & Cirripedia \\
\hline Pólipo & Naupliu \\
\hline Hidromedusas & Cypri \\
\hline \multicolumn{2}{|l|}{ Liriope tetrafila } \\
\hline \multirow[t]{2}{*}{ Calicophorae } & Mysidacea \\
\hline & Metamysidops elongata atlantica \\
\hline Mollusca & Promysis atlantica \\
\hline \multicolumn{2}{|l|}{ Veliger de bivalva } \\
\hline \multirow[t]{2}{*}{ Veliger de gastropoda } & Isopoda \\
\hline & Isopoda parasita \\
\hline \multicolumn{2}{|l|}{ Annelida } \\
\hline Larva de Polychaeta & Caprelidae \\
\hline \multicolumn{2}{|l|}{ Polychaeta (planctonico) } \\
\hline & Hiperidea \\
\hline \multicolumn{2}{|c|}{ Ctenopoda e Onychopoda (Cladocera) } \\
\hline Penilia avirostris & Euphausiacea \\
\hline Pseudevadne tergestina & Calyptopis \\
\hline \multicolumn{2}{|l|}{ Pleopsis schmarkeri } \\
\hline \multirow[t]{2}{*}{ Pleopis polyphemoides } & Decapoda \\
\hline & Ovos \\
\hline \multirow[t]{2}{*}{ Ostracoda (bentonico) } & Nauplius \\
\hline & Protozoea \\
\hline Copepoda & Mysis \\
\hline Naupliu & Zoea \\
\hline Copepodito & Megalopa \\
\hline Acartia sp & Decapodito palimura \\
\hline Acartia lilljeborgi & Decapodito de Penaeus \\
\hline Acartia tonsa & Ovo Lucifer \\
\hline Calanidae & Lucifer facione \\
\hline \multicolumn{2}{|l|}{ Calanopia americana } \\
\hline Calocalanus sp & Ctenophorae \\
\hline \multicolumn{2}{|l|}{ Centropagis velificatus } \\
\hline Corycaeus sp & Salpas \\
\hline \multicolumn{2}{|l|}{ Eucalanus pileatus } \\
\hline Euterpina acutifrons & Echinodermata \\
\hline Fhaenna spinifera & Ovo \\
\hline Harpacticoida & Pluteu \\
\hline \multicolumn{2}{|l|}{ Hemicyplops thalassius } \\
\hline Macrosetella gracilis & Chaetognatha \\
\hline Monstriloida & Sagitta sp \\
\hline Oithona sp & Sagitta enflata \\
\hline Oithona oswaldocruzii & Sagitta hispida \\
\hline Oithona plumifera & Sagitta tenuis \\
\hline \multicolumn{2}{|l|}{ Oncaea venusta } \\
\hline Paracalanus sp & Larvacea \\
\hline Paracalanus quasimodo & Oikopleura sp \\
\hline Paracalanus crassirostris & Oikopleura dioica \\
\hline \multicolumn{2}{|l|}{ Paracalanus nanus } \\
\hline Pontellidae & Doliolidae \\
\hline \multicolumn{2}{|l|}{ Pseudodiaptomus richardi } \\
\hline Temora sp & Peixes \\
\hline Temora turbinata & Ovos \\
\hline Temora stylifera & Larvas \\
\hline Copepodo bentônico & Juvenil \\
\hline Copepodo parasita & \\
\hline
\end{tabular}




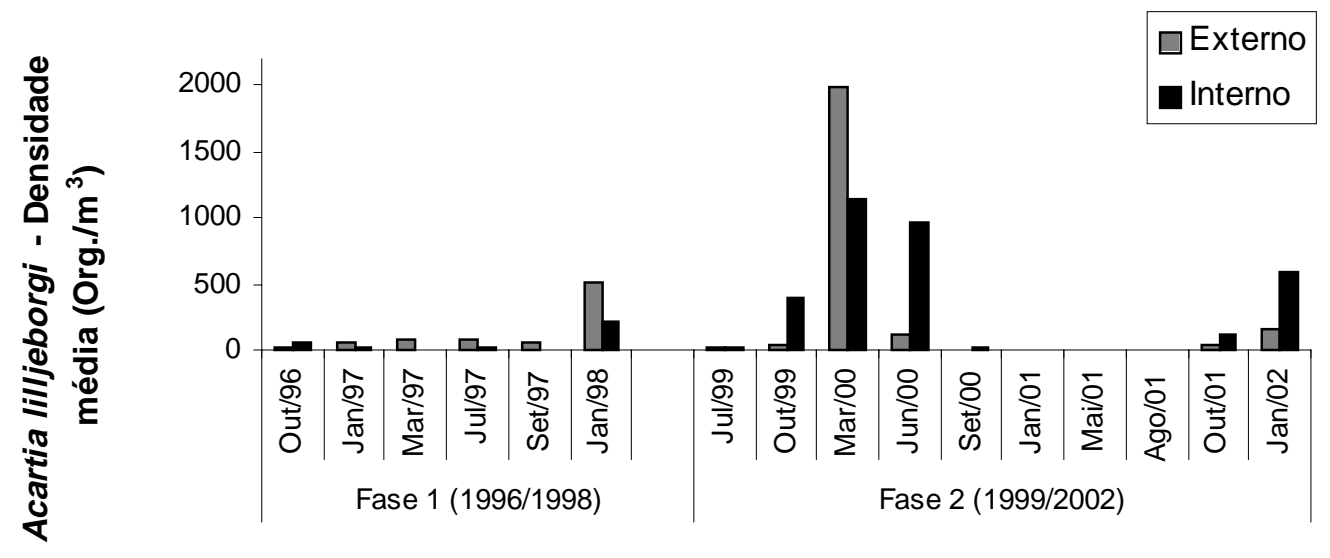

\section{Período amostral e grupos de pontos de coleta}

Figura 4 - Valores médios da densidade de Acartia lilljeborgi (Org. $/ \mathrm{m}^{3}$ ) agrupados por pontos de coleta externo e interno da enseada e por fase de estudo (Fase 1 - Impacto, Fase 2 - Pós impacto).

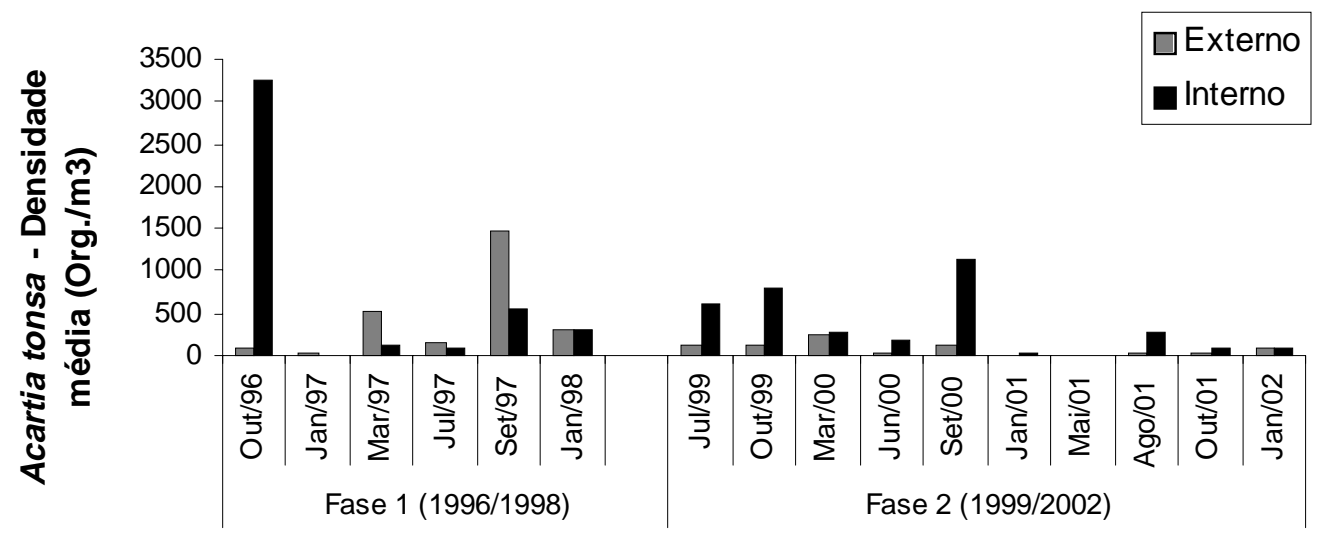

Período amostral e grupos de pontos de coleta

Figura 5 - Valores médios da densidade de Acartia tonsa $\left(\right.$ Org. $\left./ \mathrm{m}^{3}\right)$ agrupados por pontos de coleta externo e interno da enseada e por fase de estudo (Fase 1 - Impacto, Fase 2 - Pós impacto).

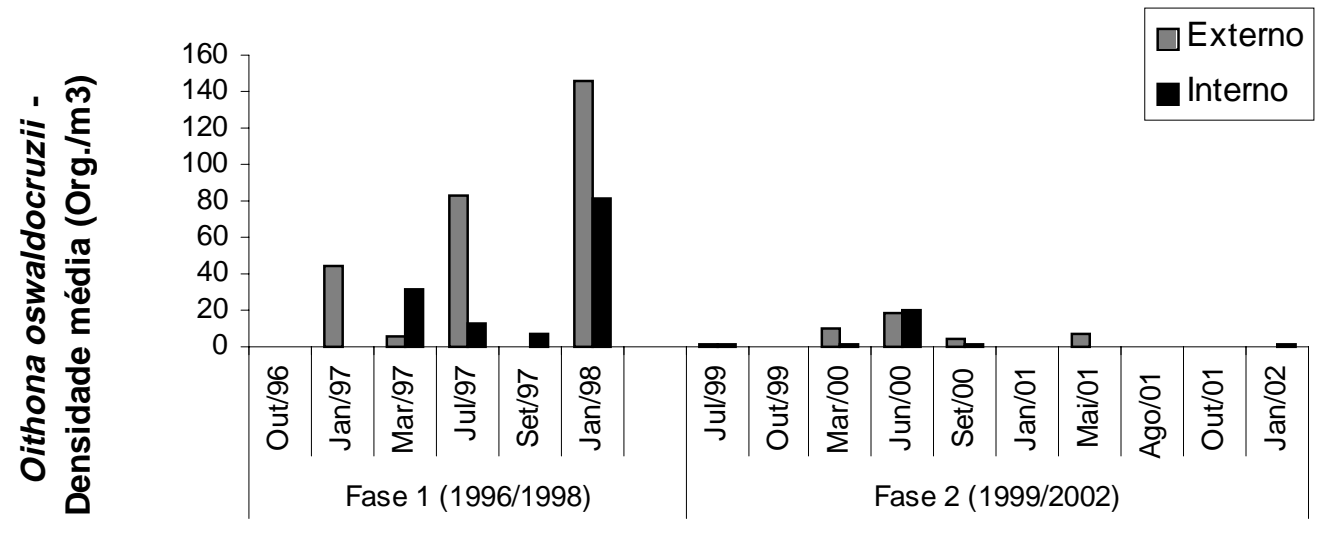

\section{Período amostral e grupos de pontos de coleta}

Figura 6 - Valores médios da densidade de Oithona oswaldocruzii (Org. $\left./ \mathrm{m}^{3}\right)$ agrupados por pontos de coleta externo e interno da enseada e por fase de estudo (Fase 1 - Impacto, Fase 2 - Pós impacto). 


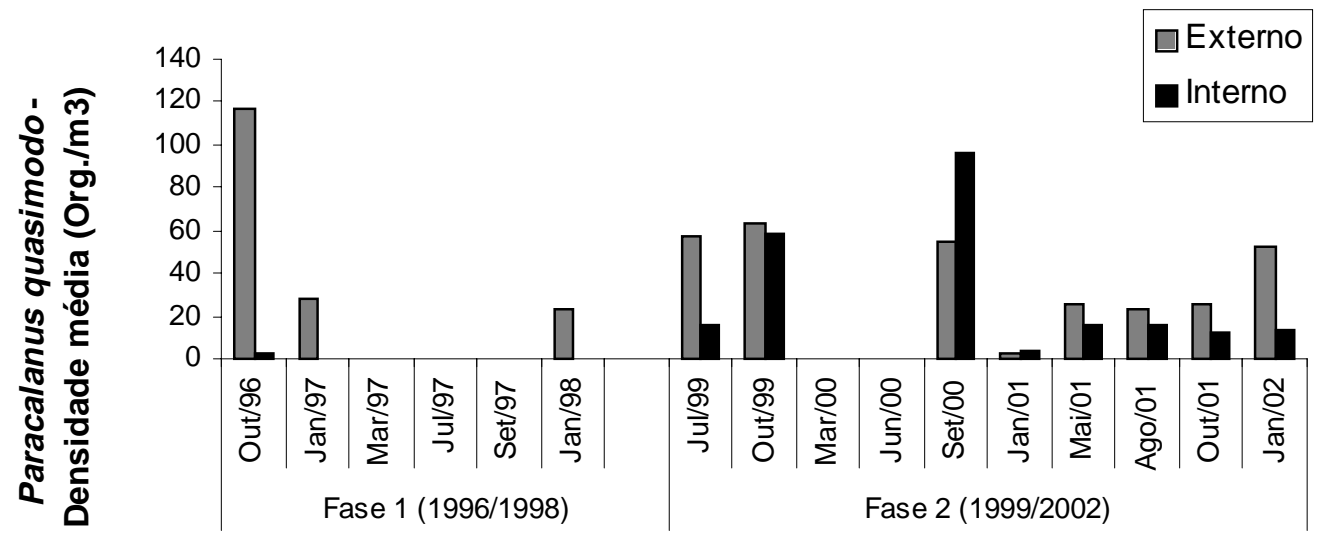

Período amostral e grupos de pontos de coleta

Figura 7 - Valores médios da densidade de Paracalanus quasimodo (Org. $/ \mathrm{m}^{3}$ ) agrupados por pontos de coleta externo e interno da enseada e por fase de estudo (Fase 1 - Impacto, Fase 2 - Pós impacto).

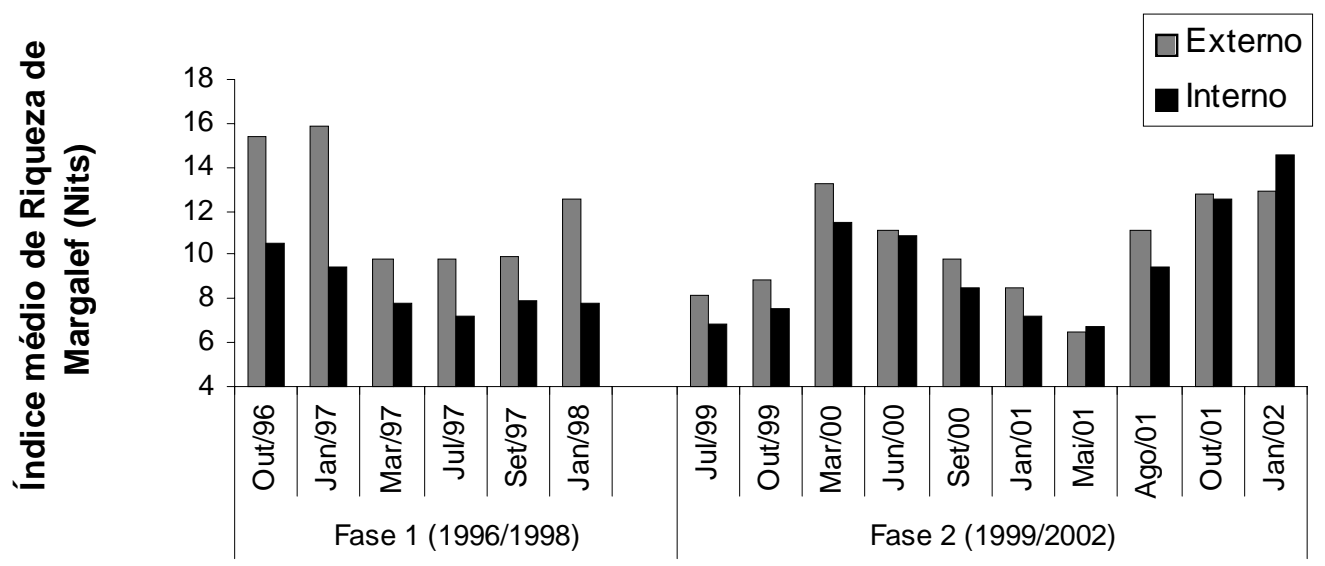

Período amostral e grupos de pontos de coleta

Figura 8 - Valores médios do índice de riqueza de Margalef agrupados por pontos de coleta externo e interno da enseada e por fase de estudo (Fase 1 - Impacto, Fase 2 - Pós impacto).

Acartia lilljeborgi é uma espécie de copépoda tipicamente costeiro, ocorrendo em águas de maiores salinidades e temperaturas (Tabela 2). Durante o período de impacto (1996/1997) esta espécie apresentou maior ocorrência fora da enseada, passando a invadir a porção interna nos anos subsequentes. Isso pôde ser constatado também para Paracalanus quasimodo que ocorreu em águas externas na primeira fase de impacto e com uma posterior invasão da região interna da enseada nos anos de pós-impacto. O contrário foi observado para Oithona oswaldocruzii que ocorreu na área estuarina da enseada em 1996/1997 e passou a uma menor representatividade nos anos seguintes.

O comportamento da Acartia tonsa ao longo do monitoramento, mostrou que a oscilação de sua densidade é concordante com a variação da densidade total do zooplâncton, podendo ser considerada como orga- nismo indicador do impacto ocorrido no ambiente. É uma espécie eurihalina, numerosa e tolerante a variações de salinidade (Cronin et al., 1962) (Tabela 2). O seu declínio no ambiente pode ser considerado como um indicativo da perda das características do Saco dos Limões, ou seja, redução no gradiente salino característico de ambientes estuarinos. Além disto, $A$. tonsa é uma espécie adaptada a altas concentrações de alimento (Reeve \& Walter, 1977 e Paffenhöfer \& Stearns, 1988), o que pode ser um fator limitante se o MS do ambiente sofreu alterações significativas. A partir de 1998, a sua dominância foi, gradativamente, sendo substituída pela $A$. lilljeborgi, que por sua vez, interferiu na ecologia pelágica do sistema (Resgalla Jr., 2001).

Além disto, a maior influência de águas costeiras na parte interna da enseada, permite uma maior distribuição de organismos zooplanctônicos carnívoros 
Veado \& Resgalla Jr.: Alteração da comunidade zooplanctônica.

Tabela 2 - Características das espécies de copépoda mais representativas da enseada do saco dos Limões (SC).

\begin{tabular}{|c|c|c|}
\hline Espécies & Características & Autor \\
\hline \multirow{2}{*}{ Acartia lilljeborgi } & Epipelágica & Bjornberg (1981) \\
& Estuário / costa & \\
\hline \multirow{2}{*}{ Acartia tonsa } & Alta temperatura & Bjornberg (1981) \\
& Estuário / costa & Montú \& Gloeden (1986) \\
& Cronin et al. (1962) \\
\hline \multirow{2}{*}{ Oithona oswaldocruzii } & Epipelágica & Bjornberg (1981) \\
\hline \multirow{2}{*}{ Paracalanus quasimodo } & Estuário / costa & Bjornberg (1981) \\
\hline
\end{tabular}

(e.g. Ctenophora, Chaetognatha, Hidromedusas, entre outros) que podem exercer forte pressão sobre a comunidade, reduzindo as densidades de espécies dominantes no ambiente (Purcell et al., 1994).

Para o Índice de Riqueza de Margalef, observouse um aumento no ambiente a partir de Janeiro de 2002, mostrando claro enriquecimento de espécies no Saco dos Limões. Apesar de ser um bom indicativo de qualidade ambiental (alto índice de riqueza), o aumento do número de espécies só vem a comprovar a diminuição do gradiente salino da enseada e, conseqüentemente, de sua característica estuarina de baixa diversidade e de alta abundância dos organismos.

\section{CONCLUSÕES}

A densidade total dos organismos apresentou um comportamento sazonal, porém sofreu um declínio após o período de impacto (1999/2002).

O zooplâncton pode ser utilizado como indicador de alterações hidrológicas, como o copépodo Acartia tonsa. A representatividade deste copépodo apresenta relação direta com a densidade total dos organismos, demonstrando as conseqüências das atividades de dragagem e aterro.

Utilizando o zooplâncton como indicador, é sugerido que a salinidade apresentou um acréscimo na região interna, permitindo a substituição da fauna estuarina por uma marinha costeira.

O Índice de Riqueza pode ser considerado como indicativo da recuperação da fauna zooplanctônica da enseada do Saco dos Limões, porém com alteração específica da comunidade. Essa alteração foi observada, principalmente, pelos copépodos Acartia lilljeborgi e $A$. tonsa, os quais apresentaram uma substituição no ambiente ao longo dos anos. A. tonsa foi substituída pela $A$. lilljeborgina região interna da enseada, indicando, provavelmente, um aumento da salinidade nesta porção e/ou devido a menor disponibilidade de alimento.

\section{AGRADECIMENTOS}

Os atores agradecem a Waldenir Inês e José Maria da Conceição pelas coletas das amostras de campo, DEER/SC pelo apoio financeiro e ao CTTMar/ UNIVALI pela viabilização dos estudos.

\section{REFERÊNCIAS}

Abreu, J. G. N., Diehl, F. I., Kuroshima, K. N., Pereira Fo., J., Santos, M. I. F., Schettini, C. A. F., Pezzuto, P. R., Rodrigues-Ribeiro, M., Hostim, M., Branco, J. O. \& Rosa, M.T. 1998. Monitoramento ambiental na área de abrangência da via expressa SC-Sul, Florianópolis, SC. Anais do 3 Encontro Ibero-Americano de Unidades Ambientais do Setor de Transportes. Florianópolis, SC, 1998. p18.

Bjornberg, T. S. K. 1981. Copepoda. In: Boltovskoy, D. (ed.), Atlas del Zooplancton del Atlantico Sudoccidental y metodos de trabajo con el zooplancton marino. Publ. Esp. INIDEPE, Mar del Plata. 587-677p. 
Boltovskoy, D. 1981a. Recuento y análisis de los datos. In: Boltovskoy, D. (ed.), Atlas del zoolplancton suddocidental y métodos de trabajo con el zooplancton marino. Publ. Esp. INIDEPE, Mar del Plata. 153-187p.

Boltovskoy, D. 1981b. Atlas del zoolplancton suddocidental y métodos de trabajo con el zooplancton marino. Publ. Esp. INIDEPE, Mar del Plata. 936pp.

Boltovskoy, D. 1999. South Atlantic Zooplankton. Backhuys Publishers, Leiden. 1706pp.

Cronin, L. E., Daiber, J.C. \& Hulbert, E.M. 1962. Quantitative Seasonal Aspects of Zooplankton in the Delaware River Estuary. Chesapeake Science, 3(2):63-93.

ENGEVIX Engenaria LTDA. 1996. Estudo, pesquisas, serviços, levantamentos, planos e programas de monitoramento, campanhas de amostragens no programa proposto e modelagem matemática, em atendimento ao IBAMA-LI, no. 02/95.
Montu, M. \& Gloeden, I.M. 1986. Atlas dos cladoceros e copépodas (Crustácea) do estuário da Lagoa dos Patos (Rio Grande, Brasil). Nerítica 1(2): 1-134.

Omori, M. \& Ikeda, T. 1984. Methods in marine zooplankton ecology. John Wiley \& Sons. 332pp.

Paffenhöfer, G.A. \& Stearns, D.E. 1988. Why is Acaria tonsa (Copepoda: Calanoida) restricted nearshore environments? Mar. Ecol. Prog. Series. 42:33-38.

Purcell, J.E., White, J.R. \& Roman, M.R. 1994. Predation by gelatinous zooplankton and resource limitation as potential controls of Acartia tonsa copepod populations in Chesapeake Bay. Limnol. Oceanogr. 29(2):263-278.

Reeve, M.R. \& Walter, M.A. 1977. Observations on the existence of lower threshold and upper critical food concentrations for the copepod Acartia tonsa Dana. J.exp.mar.Biol.Ecol. 29:211-221.

Resgalla Jr., C. 2001. Estudo de impacto ambiental sobre a comunidade do zooplâncton na enseada do Saco dos Limões, Baía Sul da Ilha de Santa Catarina, Brasil. Atlântica, 23:5-16. 